Photoelectron kinetic and angular distributions for the ionization of aligned molecules using a HHG source

This article has been downloaded from IOPscience. Please scroll down to see the full text article.

2012 J. Phys. B: At. Mol. Opt. Phys. 45074016

(http://iopscience.iop.org/0953-4075/45/7/074016)

View the table of contents for this issue, or go to the journal homepage for more

Download details:

IP Address: 194.171.111.64

The article was downloaded on 09/05/2012 at 09:25

Please note that terms and conditions apply. 


\title{
Photoelectron kinetic and angular distributions for the ionization of aligned molecules using a HHG source
}

\author{
Arnaud Rouzée ${ }^{1,2}$, Freek Kelkensberg ${ }^{2}$, Wing Kiu Siu ${ }^{2}$, \\ Georg Gademann $^{2}$, Robert R Lucchese ${ }^{3}$ and Marc J J Vrakking ${ }^{1,2}$ \\ ${ }^{1}$ Max-Born-Institut, Max-Born Straße 2A, D-12489 Berlin, Germany \\ ${ }^{2}$ FOM-Institute AMOLF, Science Park 104, 1098 XG Amsterdam, The Netherlands \\ ${ }^{3}$ Department of Chemistry, Texas A\&M University, College Station, TX 77843-3255, USA \\ E-mail: rouzee@mbi-berlin.de
}

Received 4 October 2011, in final form 15 December 2011

Published 16 March 2012

Online at stacks.iop.org/JPhysB/45/074016

\begin{abstract}
We present an experimental and theoretical investigation of the angular distributions of electrons ejected in aligned molecules by extreme ultra-violet ionization using a high harmonic generation (HHG) source. Impulsive alignment in $\mathrm{O}_{2}, \mathrm{~N}_{2}$ and $\mathrm{CO}$ molecules was achieved using a near-IR laser pulse and the photoelectron angular distribution after ionization by a fs harmonic comb composed of harmonic H11 to H29 (17.5-46 eV) was recorded at the maximum of both alignment and anti-alignment. The experiment reveals signatures that are specific for the electronic orbitals that are ionized as well as the onset of the influence of the molecular structure and is well reproduced by theoretical calculations based on the multichannel Schwinger configuration interaction method.
\end{abstract}

(Some figures may appear in colour only in the online journal)

\section{Introduction}

The development of ultrashort extreme ultra-violet (XUV) pulses by high-harmonic generation reaching into the attosecond time domain has recently opened a path towards a new class of experiments in molecular science, aimed at revealing the nature of electronic motion in molecular systems. Prior to the development of attosecond laser pulses, studies of time-dependent molecular dynamics have largely been carried out within the domain of femtochemistry. Using attosecond laser pulses, the initial stages of chemical processes, where electronic degrees of freedom couple to both the applied laser electric field and the nuclear degrees of freedom, and where the motion of multiple electrons may occur in a concerted fashion, are for the first time accessible to experimental investigation [1]. Indeed, a first application of attosecond pump-probe spectroscopy, focusing on two-colour dissociative ionization of $\mathrm{H}_{2}$ and $\mathrm{D}_{2}$ by the combination of an isolated attosecond laser pulse and a few-cycle IR pulse, was recently published [2].

The use of XUV radiation is a pre-requisite for attosecond pump-probe experiments, since only in the XUV (and
$\mathrm{X}$-ray) wavelength range does the optical period of light $(\lambda / \mathrm{c})$ permit the synthesis of attosecond light pulses. It follows that attosecond light fields necessarily consist of ionizing radiation, where the energy of individual photons may exceed molecular ionization potentials by tens or even hundreds of $\mathrm{eV}$. Thus, the photoelectrons that are ejected from molecules by these attosecond pulses can have de Broglie wavelengths that are comparable to the internuclear distances that exist in the molecule. The de Broglie wavelength of an electron is given as $\lambda_{\text {DeBroglie }}(\mathrm{au})=\pi \sqrt{2 / E_{\mathrm{kin}}(\mathrm{au})}$, where $E_{\mathrm{kin}}$ is the electron kinetic energy. It follows that a de Broglie wavelength of 1 Angström corresponds to an electron kinetic energy as low as $150 \mathrm{eV}$. Consequently in attosecond pump-probe experiments on molecular systems, attosecond time resolution may be accompanied by Angström spatial resolution.

The extraction of the complete spatial information that is encoded in the ejected photoelectrons demands that the photoelectrons are measured in the molecular frame. To date, using primarily synchrotron radiation, this has been achieved in COLTRIMS experiments, where the 3D momentum of the ejected photoelectrons is measured in coincidence with the 
3D momentum of one or more ionic fragments [3]. In the axial recoil approximation, this permits the reconstruction of the photoelectron angular distribution in the molecular frame (MFPAD). Alternatively, and preferred when using low repetition light sources like the present-day attosecond sources, which operate at repetition rates of $\leqslant 10 \mathrm{kHz}$, the photoelectron angular distribution can be obtained for samples of aligned molecules. Within the last decade, laser-induced molecular alignment has become a popular preparation step in a range of applications. Exploiting the polarization that can be induced in molecules using a moderately intense $\left(<10^{13} \mathrm{~W} \mathrm{~cm}^{-2}\right)$ laser field, both adiabatic alignment using relatively long laser pulses $\left(\tau_{\text {laser }} \gg \tau_{\text {rot }}\right)$ [4] and impulsive alignment using short laser pulses $\left(\tau_{\text {laser }} \ll \tau_{\text {rot }}\right)$ [5] can be used, the latter with the advantage that the alignment occurs under field-free conditions. Recently, laser-induced alignment techniques have been used to record the photoelectron angular distribution in strong field ionization of $\mathrm{CS}_{2}$ and benzonitrile molecules [6] and in $\mathrm{N}_{2}$ and $\mathrm{O}_{2}$ molecules [7]. Also rotationally resolved photoelectron angular distribution resulting from ionization of the $\mathrm{NH}_{3}$ molecules with optical lasers was explored recently [8].

In this paper, we present experiments where aligned samples of $\mathrm{O}_{2}, \mathrm{~N}_{2}$ and $\mathrm{CO}$ molecules were photoionized using a high harmonic generation (HHG) source. Differences between the photoelectron angular distributions that are measured for aligned and anti-aligned molecules reveal the involvement of a range of electronic states in the ionization process. Furthermore, the dependence of the photoelectron angular distributions on the high-harmonic order allows us to observe the onset of the encoding of structural information in the photoelectron angular distributions. This paper follows an earlier report that was concerned with studies of the $\mathrm{CO}_{2}$ molecule [9] for which some results are reported as well.

\section{Experimental details}

The experiments required the availability of wellsynchronized, co-propagating IR and XUV laser fields, in order to align and photo-ionize the molecules under investigation. To this end, a Mach-Zehnder interferometer was constructed where one arm provided the IR alignment laser and the other arm (which included a HHG stage) the XUV ionization laser (see figure 1). Both arms of the interferometer were fed by the output from a commercial femtosecond Ti:Sapphire amplifier (KMLabs Dragon, $3 \mathrm{kHz}, 2 \mathrm{~mJ} /$ pulse, $30 \mathrm{fs}$ ), which was split into two approximately equal parts.

HHG was performed by focusing the IR beam using a $50 \mathrm{~cm}$ focal length (FL) lens into a $3 \mathrm{~mm}$ long gas cell, containing typically 25 mbar of Ar. Phase-matching of the harmonics was accomplished by adjusting the beam size, the gas pressure and the position of the gas cell with respect to the laser focus. Following the HHG, a $200 \mathrm{~nm}$ thick aluminium filter was used to remove the generating IR light and the generated low-order harmonics $(<\mathrm{H} 11)$. The IR alignment beam and the generated high harmonics were recombined on a silver-coated drilled mirror. Subsequently the co-linearly propagating and co-polarized XUV and IR laser beams were
Table 1. Dynamic alignment of the parent molecules obtained from the fitting procedure (see the text).

\begin{tabular}{lllll}
\hline Species & $\begin{array}{l}\text { Rotational } \\
\text { constant }\left(\mathrm{cm}^{-1}\right)\end{array}$ & $\left\langle\cos ^{2} \theta\right\rangle_{\text {max,th }}$ & $\left\langle\cos ^{2} \theta\right\rangle_{\text {min,th }}$ & $\begin{array}{l}T_{\text {rot }} \\
(\mathrm{K})\end{array}$ \\
\hline $\mathrm{CO}_{2}$ & 0.39 & 0.5 & 0.2 & 75 \\
$\mathrm{O}_{2}$ & 1.436 & 0.51 & 0.19 & 40 \\
$\mathrm{CO}$ & 1.922 & 0.42 & 0.26 & 47 \\
$\mathrm{~N}_{2}$ & 1.989 & 0.45 & 0.24 & 37 \\
\hline
\end{tabular}

focused using a $40 \mathrm{~cm}$ FL toroidal mirror. The common focus of the XUV and IR beams was located in the interaction region of a velocity map imaging spectrometer (VMIS). For a given voltage, either ions or electrons are accelerated towards a twodimensional detector, consisting of two micro-channel plates (MCPs), a phosporscreen and a camera system. The XUV and IR laser beams were polarized parallel to the plane of the MCP detector, allowing a determination of the 3D velocity and angular distribution of the ejected ions and electrons from the measured projections, using an Abel-inversion routine based on a Legendre polynomial expansion. The samples under investigation were introduced by means of a pulsed valve that was integrated into the repeller electrode [10].

In figure 2, the angular distributions of $\mathrm{O}^{+}$and $\mathrm{N}^{+}$fragments are plotted that result from $\mathrm{XUV}$-induced ionization and dissociation of $\mathrm{CO}_{2}, \mathrm{O}_{2}, \mathrm{~N}_{2}$ and $\mathrm{CO}$ molecules, as a function of the time delay between the impulsive alignment by the IR laser and the XUV pulse. In previous measurements [11], we have shown the possibility of using the fragments resulting from the dissociative ionization of the molecule by an XUV pulse to quantify the degree of molecular alignment. If the dissociation occurs on a timescale shorter than the rotational period of the molecule, the angular distribution of the ejected fragments follows the IR laser-induced alignment dynamic. Indeed, figure 2 shows the occurrence of an IRlaser-induced alignment shortly after the excitation by the IR laser pulse, which is followed by a series of alignment revivals which occur at regular intervals determined by the rotational constants of the molecules under investigation (see table 1). Transient alignment occurs because the ultrashort laser pulse imparts a kick on a timescale that is short compared to the rotational period (i.e. $\tau_{\text {laser }} \ll \tau_{\text {rot }}$ ). In this limit, the interaction leads to the creation of a rotational wave packet that evolves under field-free conditions once the alignment laser field has ended. Due to the quantization of the rotational energy levels, the free evolution of this superposition of states leads to a recurring alignment and anti-alignment of the molecules due to the rephasing of the rotational components. Figure 3 shows the experimental expectation values $\left\langle\cos ^{2} \theta_{2 \mathrm{D}}\right\rangle$ with $\theta_{2 \mathrm{D}}$ the polar angle between the alignment laser polarization axis and the recoil direction of the fragment ion. The red line in figure 3 shows the calculated $\left\langle\cos ^{2} \theta\right\rangle$, with $\theta$ the angle between the molecular axis and the laser polarization axis, that is obtained by solving the time-dependent Schrödinger equation (TDSE) for a sample of molecules interacting with a strong laser field. A least-squares method was used to reproduce the experimental result using the temperature of the molecules and the intensity of the alignment laser pulse as 

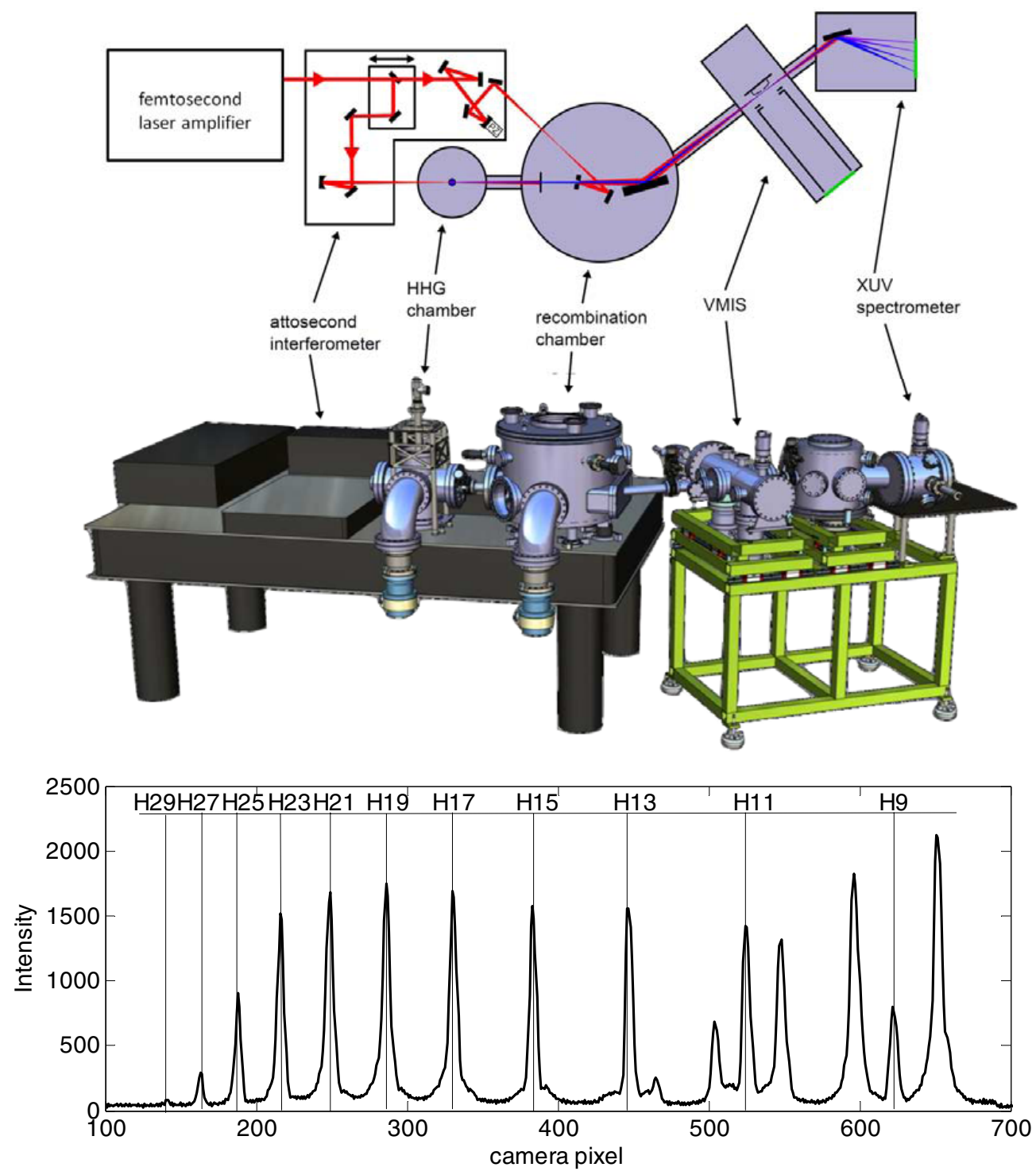

Figure 1. Top: the attosecond pump-probe setup at AMOLF. The output of the Ti:Sa laser is split into two beams that form the two arms of a Mach-Zehnder interferometer. In one arm, the laser is focused into a HHG gas cell. Following the HHG process, the remaining IR light and the generated low-order harmonics are removed by means of a filter. This arm is then recombined with the other arm in the recombination chamber. The co-linearly propagating XUV and IR beams are brought to a common focus in the centre of a VMIS by using a toroidal mirror. In the VMIS, charged particles (electrons or ions) resulting from the interaction with the XUV and IR laser pulses are projected onto a two-dimensional detector. Finally, an XUV spectrometer that follows the VMIS monitors the harmonic spectrum (bottom). The unassigned lines in the XUV spectrum come from high-order diffracted light.

fitting parameters. Also the theoretical curve is given an offset to account for the geometrical alignment that results from the angular dependence of the photoionization by the XUV pulse. The result of the fitting procedure is given in table 1 . The degree of alignment achieved in this study is smaller than what could be achieved due to the modest degree of rotational cooling of the molecules that exit the pulsed valve and the relatively low intensity of the alignment laser, which was used in order to avoid ionization of the molecules.

Ionization from the XUV pulse leads to the observation of a large number of rings in the photoelectron velocity map images (see figure 4 for a measurement obtained for 'XUVonly' ionization of $\mathrm{O}_{2}$ ). This large number of rings is caused by the fact that the high-harmonic beam contains multiple harmonic orders that can ionize (odd harmonic orders spanning
H11-H29, corresponding to photon energies between 17 and $46 \mathrm{eV}$ ). Moreover, the ionization by each harmonic order leads to the production of a distinct number of peaks in the velocity distribution of the produced electrons, due to the fact that the XUV light removes electrons from valence and inner-valence orbitals. In the case of $\mathrm{O}_{2}$ that is shown in figure 4 , for example, contributions from as many as five orbitals can be identified in the measured XUV photoelectron velocity map image. Similar experiments using synchrotron radiation or electron impact have shown that the photoelectron spectra contains up to eight contributions $[13,14]$.

In order to assess the role of molecular alignment, photoelectron velocity map images were recorded at selected delays between the IR alignment laser and the XUV ionization laser. These delays were chosen to coincide with time delays 
(a) $\mathrm{CO}_{2}$

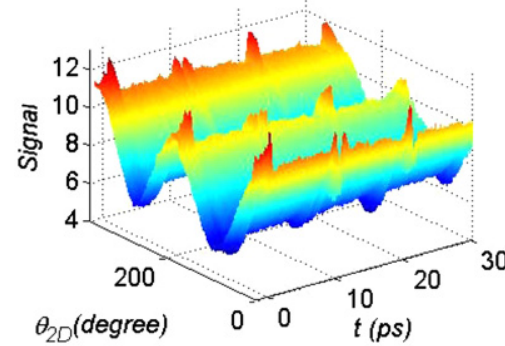

(c) $\mathrm{CO}$

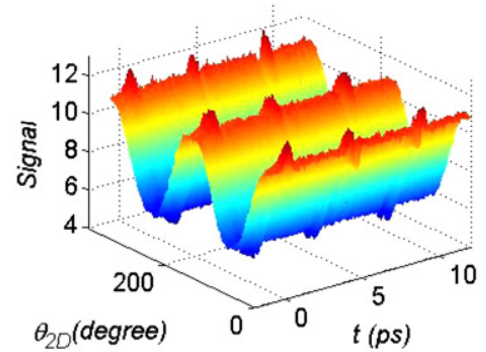

(b) $\mathrm{O}_{2}$

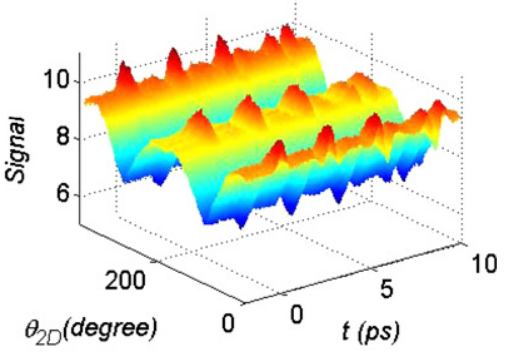

(d) N2

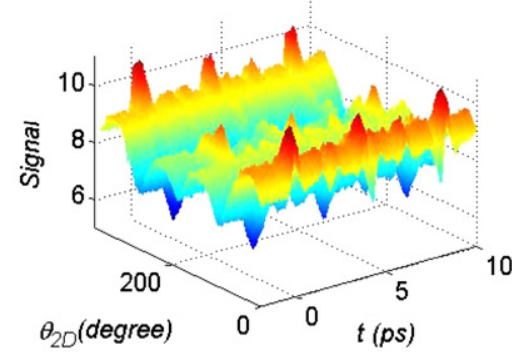

Figure 2. Time evolution of the angular distributions measured in XUV-induced Coulomb explosion, as a function of the time delay between the IR laser that impulsively aligns the molecules and the $\mathrm{XUV}$ pulse that Coulomb explodes the molecule for (a) $\mathrm{CO}_{2}$, (b) $\mathrm{O}_{2}$, (c) $\mathrm{CO}$ and (d) $\mathrm{N}_{2}$. In the axial recoil approximation, the measured fragment ion angular distributions allow us to determine the molecular alignment $\left\langle\cos ^{2} \theta_{2 \mathrm{D}}\right\rangle$ as a function of time delay. In the present graphs, the occurrence of alignment and anti-alignment can be recognized at specific time delays.

(a) $\mathrm{CO}_{2}$

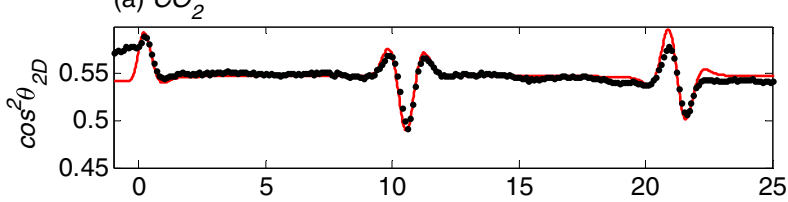

(b) $\mathrm{O}_{2}$

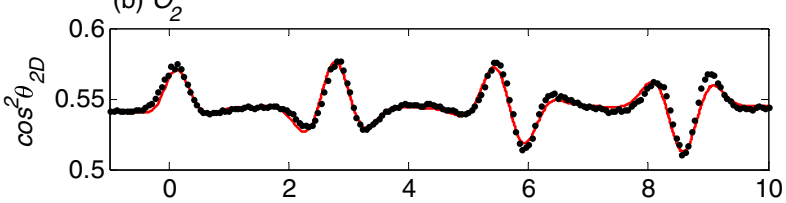

(c) $\mathrm{CO}$

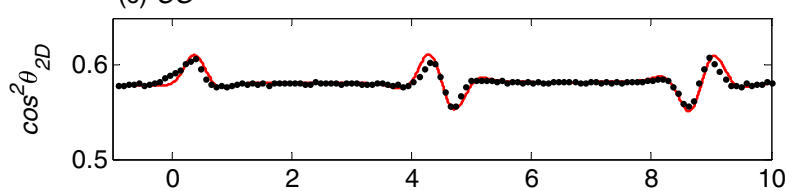

(d) $N_{2}$

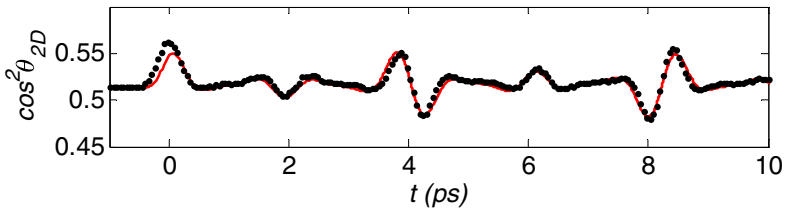

Figure 3. (a)-(d) Experimental (black dots) and theoretical (red lines) alignment of $\mathrm{CO}_{2}, \mathrm{O}_{2}, \mathrm{CO}$ and $\mathrm{N}_{2}$, as a function of the time delay between the IR alignment laser and the XUV ionization laser. The numerical calculations are based on the method described in [12] and allow a determination of the rotational temperature of the molecular sample and the average intensity of the IR beam in the XUV focus (see table 1).

where the ionic fragments were produced maximally aligned along the alignment laser polarization axis, or maximally perpendicular to the alignment laser polarization axis ("planar delocalization'). Typical acquisitions consisted of taking data for a total of $1.2 \times 10^{6}$ laser shots at both delays. At first sight (see figure 5(a)), the photoelectron images recorded at both time delays look very similar. However, given the high signalto-noise of both images, differences can be observed with good statistics between the images that are recorded for aligned and anti-aligned molecules. This is illustrated in figure 5(b), which shows a difference of photoelectron velocity map images, i.e. the difference between a slice through the reconstructed 3D photoelectron momentum distribution for the aligned and the anti-aligned $\mathrm{O}_{2}$ samples. In the difference of velocity map images, like in the XUV photoelectron velocity map images, a large number of rings can be observed, corresponding to ionization of a particular orbital of the molecule by a particular harmonic. In particular, a clear correlation between the orbital from which the electron is removed and the differential angular distribution can be identified. A detailed discussion of this correlation and a comparison of the experimental results to numerical calculations will be presented below.

\section{3. $\mathrm{O}_{2}$}

In order to discuss the results obtained for $\mathrm{O}_{2}$, we first discuss the decomposition of the XUV photoelectron spectrum (figure 4) into contributions from different combinations of the ionizing orbital and the harmonic order. The photoelectron velocity map image contains dominant contributions from ionization of the HOMO $\left(\mathrm{X}^{2} \Pi_{\mathrm{g}}\right)$ and HOMO-1 $\left(\mathrm{a}^{4} \Pi_{\mathrm{u}}\right)$ and other contributions from ionization of the HOMO-2 $\left(\mathrm{A}^{2} \Pi_{\mathrm{u}}\right)$, HOMO-3 ( $\left.\mathrm{b}^{4} \Sigma^{-}{ }_{\mathrm{g}}\right)$ and HOMO-4 ( $\left.\mathrm{B}^{2} \Sigma^{-}{ }_{\mathrm{g}}\right)$. Most of these contributions can be recognized in the two photoelectron spectra that are shown on the right side of figure 4 , which 


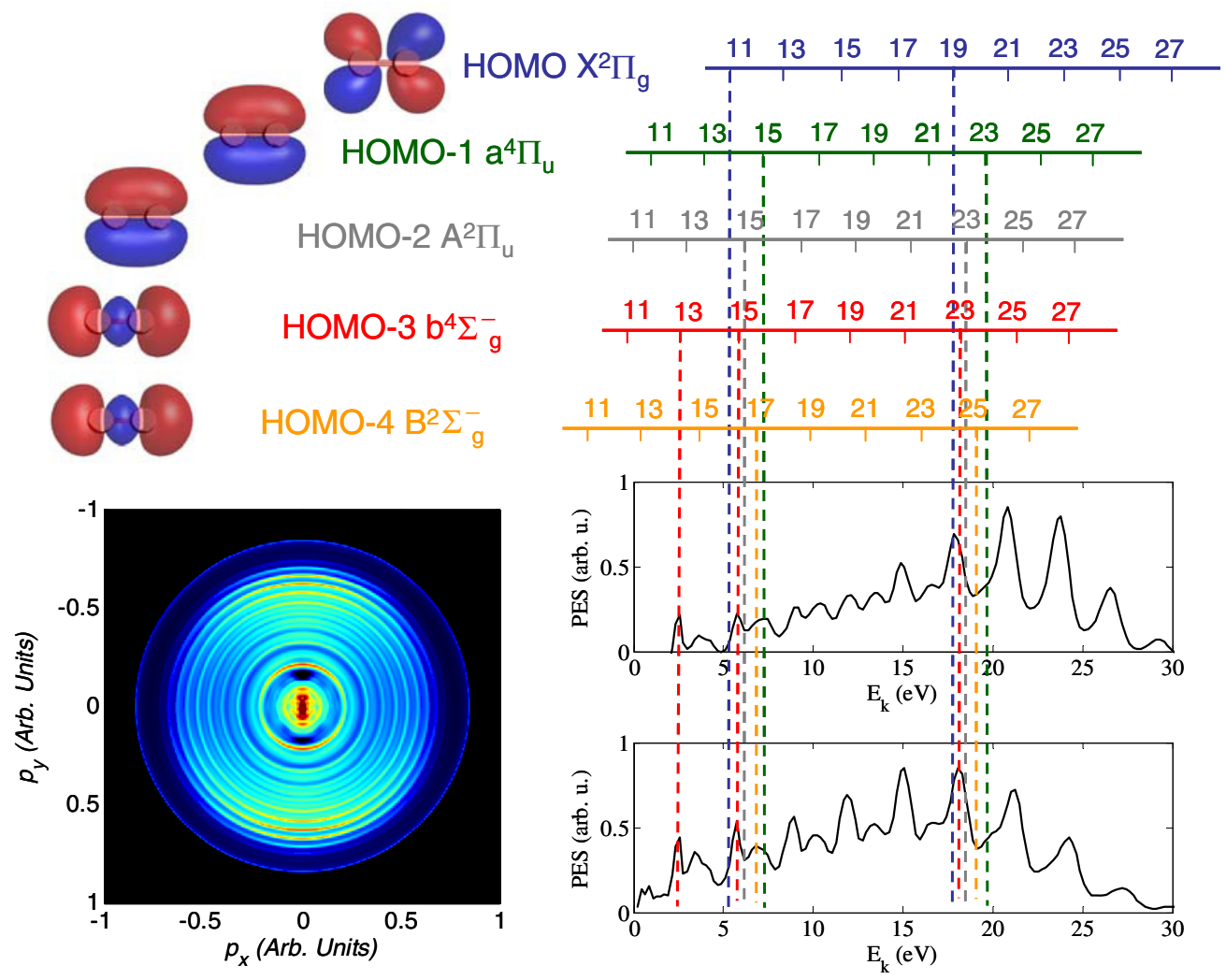

Figure 4. Ionization of $\mathrm{O}_{2}$ by the XUV pulse, involving contributions from harmonics H11-H27. The image in the bottom left represents a 2D slice through the 3D velocity and angular distribution of the ejected photoelectrons and shows a large number of rings that can be assigned to contributions from the ionization of particular orbitals by a selected harmonic. The line-outs on the right show two photoelectron spectra obtained from the 2D slice by considering only the photoelectrons ejected along the vertical (top) and horizontal (bottom) axes. Assignments corresponding to ionization from the HOMO to HOMO-4 orbital of the neutral molecule by selected harmonics are indicated at the top.

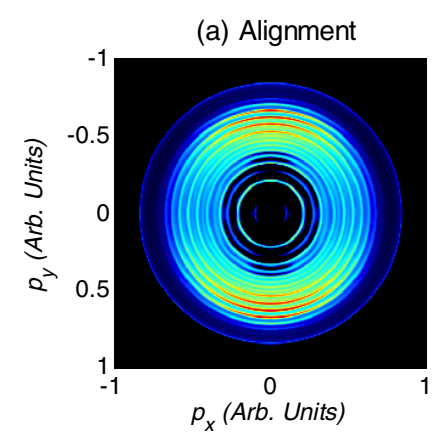

(b) Anti-alignment

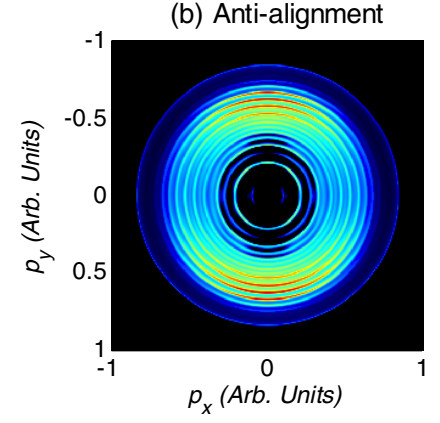

(c) Alignment-Antialignment

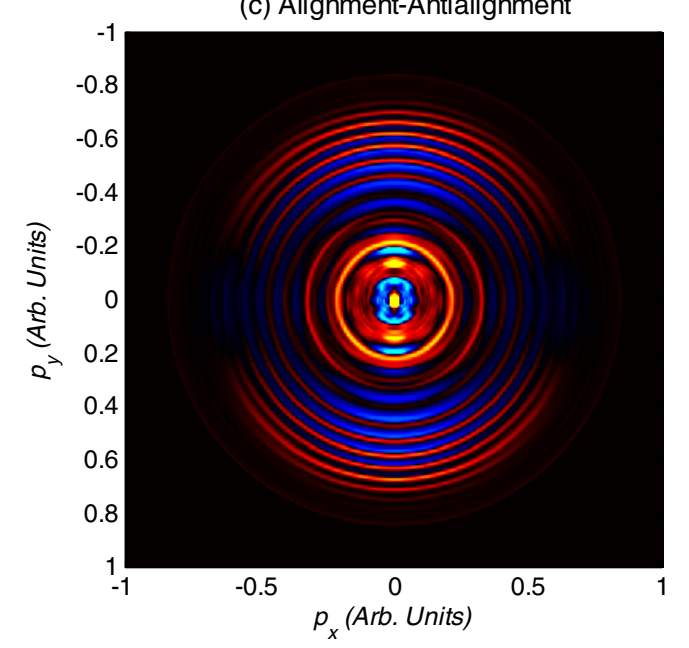

Figure 5. (a), (b) 2D slices through the 3D velocity and angular distribution of photoelectrons ejected by XUV ionization from aligned, resp. anti-aligned $\mathrm{O}_{2}$ molecules; (c) photoelectron image calculated as the difference between the images shown in (a) and (b), and illustrating that the XUV photon energy and the orbital from which the photoelectron is removed together leave a specific signature. Contributions that are red signify contributions that are more likely aligned, as opposed to anti-aligned molecules, whereas blue contributions favour anti-aligned molecules. 
were obtained from the $2 \mathrm{D}$ slice through the $3 \mathrm{D}$ velocity map image by considering only the photoelectrons ejected along the vertical (top) and horizontal (bottom) axes. In the case of single photon ionization, the kinetic energy of the outgoing electron is given by

$$
E_{\mathrm{ke}}=h v-E_{\mathrm{ion}}^{i},
$$

where $E_{\mathrm{ion}}^{i}$ is the energy difference between the cationic state and the ground state, which in the case of $\mathrm{O}_{2}$ is given by $12.3,16.7,17.6,18.2$ and $20.3 \mathrm{eV}$ for the $\mathrm{X}^{2} \Pi_{\mathrm{g}}, \mathrm{a}^{4} \Pi_{\mathrm{u}}, \mathrm{A}^{2} \Pi_{\mathrm{u}}$, $\mathrm{b}^{4} \Sigma^{-} \mathrm{g}$ and $\mathrm{B}^{2} \Sigma^{-} \mathrm{g}$ states, respectively. The decomposition of the photoelectron spectrum into contributions from these orbitals will become even clearer below, when the interpretation of the difference of photoelectron velocity map images is discussed.

As mentioned above, a photoelectron velocity map image was obtained by taking the difference between two images resulting from a total of $1.2 \times 10^{6}$ laser shots each, where photoelectrons were recorded under conditions (i) where the parent molecules were maximally aligned $\left(\Delta t_{\mathrm{IR}-\mathrm{XUV}}=\right.$ $5.4 \mathrm{ps}$ ) and (ii) where the parent molecule was in a condition of planar delocalization $\left(\Delta t_{\mathrm{IR}-\mathrm{XUV}}=5.9 \mathrm{ps}\right)$. The resulting difference between the photoelectron velocity map images is plotted in figures 5 and 6(a), where the display in Cartesian coordinates $\left(p_{x}, p_{y}\right)$, with $p_{x}$ and $p_{y}$ the momentum of the ejected electrons perpendicular and along the laser polarization axis, is replaced by a representation where the photoelectron intensity is plotted as a function of the photoelectron kinetic energy and the polar angle (between 0 and $\pi / 2$ ) of the photoelectron velocity with respect to the polarization axis of the ionizing XUV laser. In figure 6(a), a comparison with results from calculations based on the multichannel Schwinger configuration interaction method (MCSCI) is included. Such calculations have previously been successfully applied to calculate MFPADs that were recorded with synchrotron radiation. In this method, the initial state and the final ionic states are represented as configuration interaction (CI) wavefunctions [15-18]. All calculations were performed at the experimental equilibrium geometry with $\mathrm{R}(\mathrm{O}-\mathrm{O})=1.20752$ Angström. The one-electron basis set, used to construct the bound state wavefunctions, was the augmented correlationconsistent polarized valence triple- $\zeta$ basis set (aug-cc-pVTZ) $[19,20]$. The bound orbitals used in both the initial and final states were computed using valence complete active space selfconsistent field (CASSCF) calculations on the initial neutral molecule. All bound state calculations were performed using the MOLPRO quantum chemistry code. All integrals were performed using a single-centre expansion (SCE) including partial waves up to $l_{\max }=80$. The five outer valence ion states of $\mathrm{O}_{2}{ }^{+}$that were found to contribute to the experimental data $\left(X^{2} \Pi_{g}, a^{4} \Pi_{u}, A^{2} \Pi_{u}, b^{4} \Sigma^{-}\right.$g and $B^{2} \Sigma^{-}$g) were included in the MCSCI calculation. The ionization potentials of these states were adjusted so that they corresponded to the experimental vertical ionization potential. This adjustment ensures that the photoelectron kinetic energy will have the correct asymptotic value, which is necessary since the photoelectron angular distribution is sensitive to the photoelectron kinetic energy. In the calculations, the CI wavefunction was not the full valence CI wavefunction. We have limited the excitation level to at most five electrons in the weakly occupied orbitals in the initial state wavefunctions and four electrons in the weakly occupied orbitals in the ion state wavefunctions. This kept the CI wavefunctions from getting too large, with no more than 6852 configuration state functions in the ion state wavefunctions. Once the wavefunctions are computed, the transition matrix elements and the corresponding MFPAD were calculated for different angles between the molecular axis and the laser polarization axis. The laboratory frame photoelectron angular distributions (LFPADs) were obtained by averaging over the angular distribution of the molecular axis that was measured experimentally (see figure 2) in the case of maximum of alignment and antialignment. A difference between the two calculated LFPADs is then performed. Finally, to simulate the experimental results, the harmonic spectrum was written as

$$
I\left(E_{\mathrm{XUV}}\right)=\Sigma_{n} a_{n} \exp \left(-4 \log (2)\left[\left(n E_{\mathrm{IR}} / w_{\mathrm{XUV}}\right]^{2}\right)^{2},\right.
$$

with $n$ the harmonic order, $E_{\mathrm{IR}}$ the photon energy of the IR laser that generates the high-harmonics, $w_{\mathrm{XUV}}$ the spectral width (FWHM) of the individual harmonics and $a_{n}$ the amplitude of the $n$th harmonic. $w_{\mathrm{XUV}}$ and $a_{n}$ were determined by fitting the experimental results at $\theta_{\mathrm{e}-}=0, \pi / 3$ and $\pi / 2$.

The experimental difference of photoelectron angular distributions is shown in figure 6(a). Each feature that is observed in figure 6(a) can be assigned to a particular ionization channel and a particular harmonic order and can be separated using expression (1). Figure 6(a) contains characteristic sequences of positive and negative contributions that are well reproduced by the calculations. Ionization to the $\mathrm{X}^{2} \Pi_{\mathrm{g}}$ leads for instance to a positive contribution along the laser polarization axis (see figure 6(b)) whereas ionization resulting in the final ionic states $a^{4} \Pi_{u}$ and $A^{2} \Pi_{u}$ has a negative contribution with a pronounced dip along and perpendicular to the laser polarization (see figures 6(c) and (d)).

Electrical dipole transitions from the neutral ground state of $\mathrm{O}_{2}$ with $\mathrm{X}^{3} \Sigma^{-}$g symmetry restrict the total symmetry of the photoelectron + remaining cationic states to $\Sigma_{\mathrm{u}}$ for a parallel transition and $\Pi_{\mathrm{u}}$ for a perpendicular transition, and manifest as a positive (negative) sign in the difference of PADs. In other words, parallel transitions take place preferably in molecules aligned parallel to the laser polarization axis, leading to a positive difference of photoelectron yields, whereas perpendicular transitions favour molecules that are aligned orthogonally to the laser polarization axis, making the difference of photoelectron yields negative. It follows that in the present case, the ionization from the $\mathrm{X}^{3} \Sigma^{-} \mathrm{g}$ state to the $\mathrm{X}^{2} \Pi_{\mathrm{g}}$ state is mainly parallel, whereas transitions from the $\mathrm{X}^{3} \Sigma^{-} \mathrm{g}$ state to either the $\mathrm{a}^{4} \Pi_{\mathrm{u}}$ or $\mathrm{A}^{2} \Pi_{\mathrm{u}}$ ionic states have mainly a perpendicular character in this photon energy range. Also the change from positive to negative behaviour observed around $12 \mathrm{eV}$ in the $\mathrm{b}^{4} \Sigma^{-}{ }_{\mathrm{g}}$ and $\mathrm{B}^{2} \Sigma^{-}{ }_{\mathrm{g}}$ channels (see figures 6(e) and (f)) reflects a switch of the final state symmetry from $\Sigma_{\mathrm{u}}$ to $\Pi_{\mathrm{u}}$, which necessarily means a change in the symmetry of the outgoing electron. One can quantify the importance of parallel and perpendicular transition using the expression

$$
\beta=\frac{2 I_{/ /}-I_{\perp}}{I_{/ /}+2 I_{\perp}},
$$




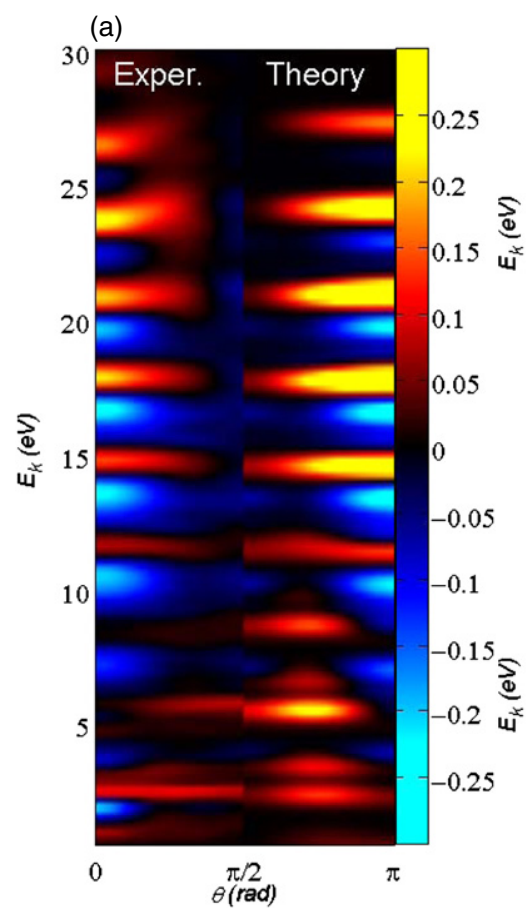

(b) $x^{2} \Pi_{g}$

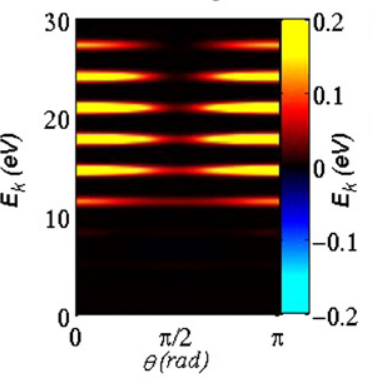

(e) $b^{4} \Sigma_{g}$

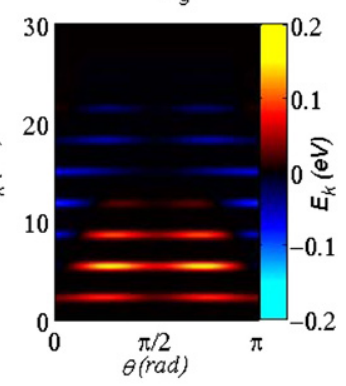

(c) $a^{4} \Pi_{u}$

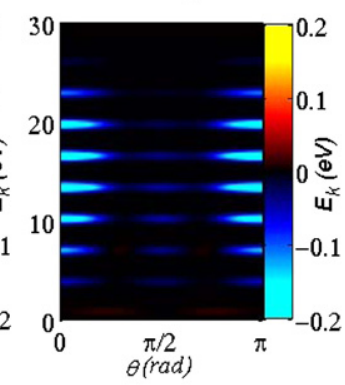

(f) $B^{2} \Sigma_{g}$

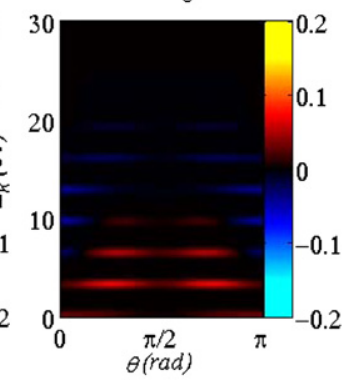

(d) $A^{2} \Pi_{u}$

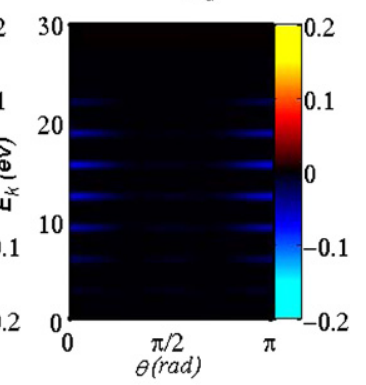

$\theta(\mathrm{rad})$

Figure 6. Difference of photoelectron angular distributions obtained after subtracting the PAD recorded at the maximum of alignment and anti-alignment in $\mathrm{O}_{2}$ molecules. (b)-(f) The calculated evolution of the differential PAD with the kinetic energy for the five ionization channels observed in the experiment.

where $I_{/ /}$and $I_{\perp}$ are the ionization efficiency of the molecule by a laser field parallel and perpendicular to the molecular axis, respectively. A perfectly parallel transition leads to $\beta=2$ whereas a perfectly perpendicular transition leads to $\beta=-1$. The rather broad angular distribution of the aligned molecular sample in our experiment leads to a positive $\beta$ contribution for all five ionization channels, which is also reproduced theoretically when taking this molecular axis alignment distribution into account. However, calculations performed for the case of perfect alignment and anti-alignment show a positive $\beta$ for the $X^{2} \Pi g$ channel ( $\beta=1$ for H17) and a negative $\beta$ for the $\mathrm{a}^{4} \Pi_{\mathrm{u}}$ and $\mathrm{A}^{2} \Pi_{\mathrm{u}}$ channels $(\beta=-0.16$ and -0.22 for $\mathrm{H} 17$, respectively). Ionization leading to the final $\mathrm{b}^{4} \Sigma^{-}{ }_{\mathrm{g}}$ and $\mathrm{B}^{2} \Sigma^{-} \mathrm{g}$ ionic states has a $\beta$ parameter that switches from a positive value ( $\beta=0.87$ and 0.88 respectively for H17) to a negative value $(\beta=-0.08$ and -0.25 for harmonic 21). These tendencies are well reproduced in the experimentally recorded difference of PADs.

The wavefunction of a photoelectron ejected by single photon ionization can be expressed as a superposition of partial waves that are characterized by the angular momentum $l$ of the photoelectron and a symmetry index $\lambda$ that describes the projection of this angular momentum on the molecular axis; for $\lambda=0$, the states are designated as $\sigma$ and for $\lambda=1$ as $\pi$. In practice, the partial-wave expansion converges at relatively small $l(l<4$, or in usual notation $\mathrm{s}, \mathrm{p}, \mathrm{d}$ and $\mathrm{f}$ partial waves). In addition, the dipolar $u \leftrightarrow g$ selection rule restricts the electron in the continuum to ungerade symmetry for the final ionic states $\mathrm{X}^{2} \Pi_{\mathrm{g}}, \mathrm{b}^{4} \Sigma^{-} \mathrm{g}$ and $\mathrm{B}^{2} \Sigma^{-} \mathrm{g}$ (which implies that only odd angular momenta appear in the partial wave expansion of the outgoing photoelectron) and gerade symmetry for the $a^{4} \Pi_{u}$ and $A^{2} \Pi_{u}$ states of $\mathrm{O}_{2}$ (which implies that only even angular momenta appear in the partial wave expansion of the outgoing photoelectron). One cannot extract the partial wave decomposition of the electronic wave packet from the experimental results due to the low degree of alignment. However, the experimental result does put considerable constraints on the possible decomposition of the electronic wavepacket into partial waves. For the ionization leading to the final $X^{2} \Pi_{g}$ ground ionic state for instance, where the wave packet is mainly composed of $l=1,3$ ( $\mathrm{p}$ and $\mathrm{f}$ waves), the pronounced positive contribution along the laser polarization axis that is observed both theoretically and experimentally (figure 6(b)) suggests that the photoelectron partial wave decomposition is dominated by a contribution from the p $\pi_{u}$ partial wave. For the $\mathrm{a}^{4} \Pi_{\mathrm{u}}$ and $\mathrm{A}^{2} \Pi_{\mathrm{u}}$ states, the difference of PADs has a strong negative signal with pronounced dips along and perpendicular to the laser polarization (figures 6(c) and (d)) since the ejected electron is dominated by a d-wave component $(l=2)$. As already mentioned, ionization to these two ionic states proceeds mainly via a perpendicular transition. The outgoing electron is therefore expected to have a strong $\mathrm{d} \sigma_{\mathrm{g}}$ component. Finally, for the two last ionic states observed in our experiment, i.e. $\mathrm{b}^{4} \Sigma^{-}{ }_{\mathrm{g}}$ and $\mathrm{B}^{2} \Sigma^{-}{ }_{\mathrm{g}}$, the difference of PADs shows a strong component along and at $60^{\circ}$ of the laser polarization axis which suggests that the partial wave decomposition of the electron wave packet is composed of $l=1$ (i.e. p-wave) and $l=3$ (i.e. f-wave). Considering as well the change in the sign of the difference of PADs, this indicates that the electron wave packet is composed of a $\mathrm{p} \sigma_{\mathrm{u}}$ and f $\sigma_{\mathrm{u}}$ component at low kinetic energy (below $12 \mathrm{eV}$ ) that changes to a $\mathrm{p} \pi_{\mathrm{u}}$ and $\mathrm{f} \pi_{\mathrm{u}}$ component at higher kinetic energy. For all five channels, modifications of the angular distribution of the ejected photoelectron are observed when increasing the 
(a)

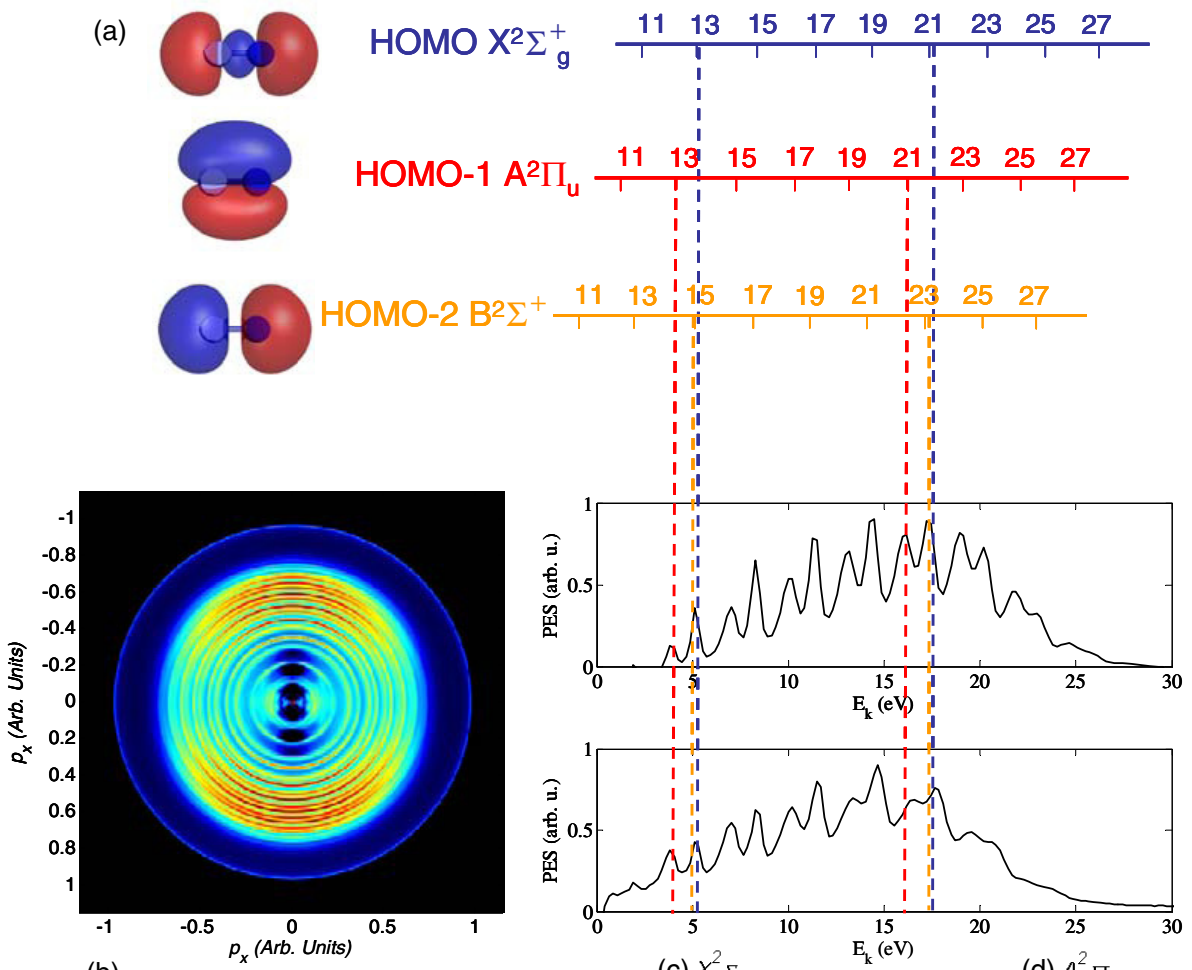

(b)

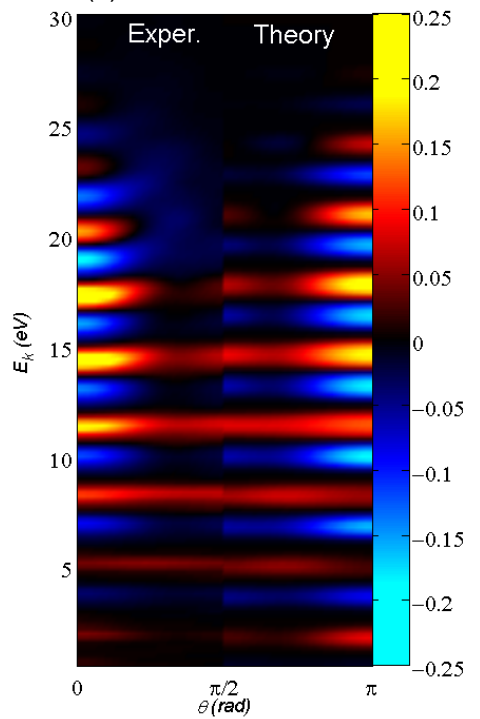

(c) $x^{2} \Sigma_{g}$
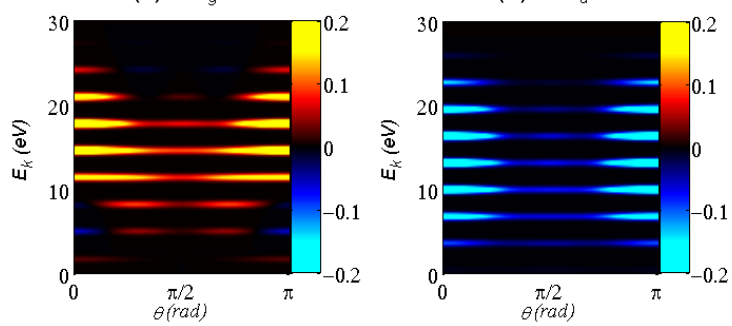

(e) $B^{2} \Sigma_{u}{ }^{+}$

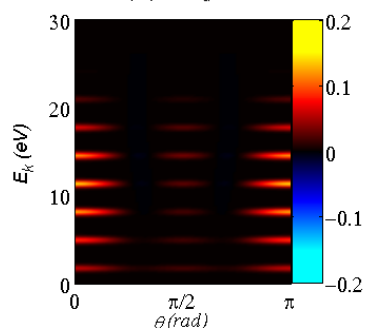

Figure 7. (a) Ionization of $\mathrm{N}_{2}$ by the XUV pulse, involving contributions from harmonics H11-H27. The bottom-left panel shows the retrieve $2 \mathrm{D}$ slice through the $3 \mathrm{D}$ electron momentum distribution obtained after inversion of the experimental data. The bottom-right panel shows the integrated photoelectron spectra along (top) and perpendicular (bottom) to the laser polarization. (b) Angular and kinetic energy distribution of the difference of PADs obtained in aligned and anti-aligned $\mathrm{N}_{2}$ molecules and comparison with theory. (c)-(e) The calculated evolution of the difference of PADs with respect to the kinetic energy for the three ionization channels observed in the experiment.

kinetic energy. These changes are related to the different partial waves that compose the electronic wave packet. Partial waves have different Coulomb phase shift with their own energy dependences. From a general point of view, the modification of the PAD with kinetic energy reflects the increased sensitivity of the electron to the molecular (Coulomb) field. The effect of the Coulomb field on the outgoing electron depends on its kinetic energy and affects the coupling between the partial waves, leading to the observed evolution of the angular distribution with kinetic energy.

\section{4. $\mathrm{N}_{2}$}

In $\mathrm{N}_{2}$, the photoelectron spectra are dominated by three contributions leading to the formation of the $\mathrm{X}^{2} \Sigma^{+}{ }_{\mathrm{g}}(15.6 \mathrm{eV})$, $\mathrm{A}^{2} \Pi_{\mathrm{u}}(17 \mathrm{eV})$ and $\mathrm{B}^{2} \Sigma^{+}{ }_{\mathrm{u}}(18.8 \mathrm{eV}$ ) ionic states [21, 22] (see figure 7(a)), corresponding to the removal of an electron from the HOMO, HOMO-1 and HOMO-2 orbitals. The difference in the binding energy between the $\mathrm{X}^{2} \Sigma^{+}{ }_{\mathrm{g}}$ and $\mathrm{B}^{2} \Sigma^{+}{ }_{\mathrm{u}}$ states corresponds almost exactly to the difference in photon energy between two consecutive harmonics. Consequently, these two 
(a)
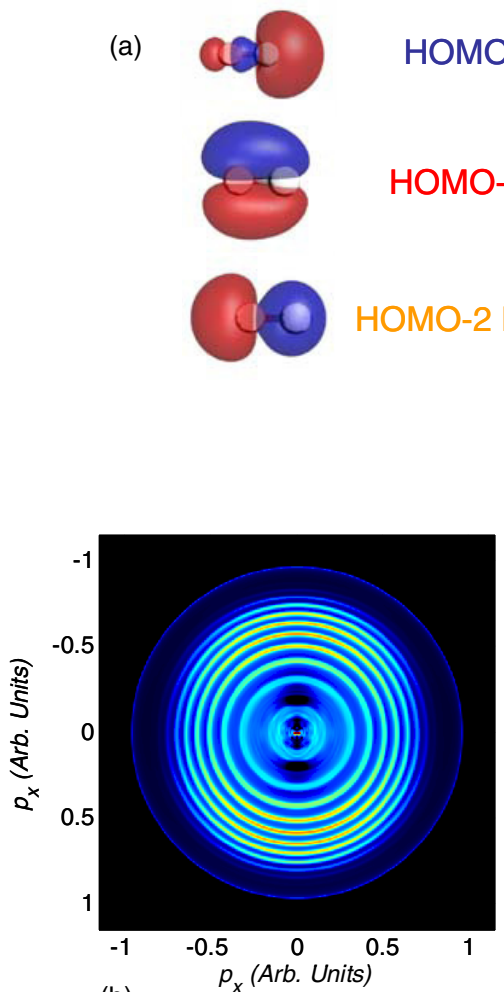

(b)

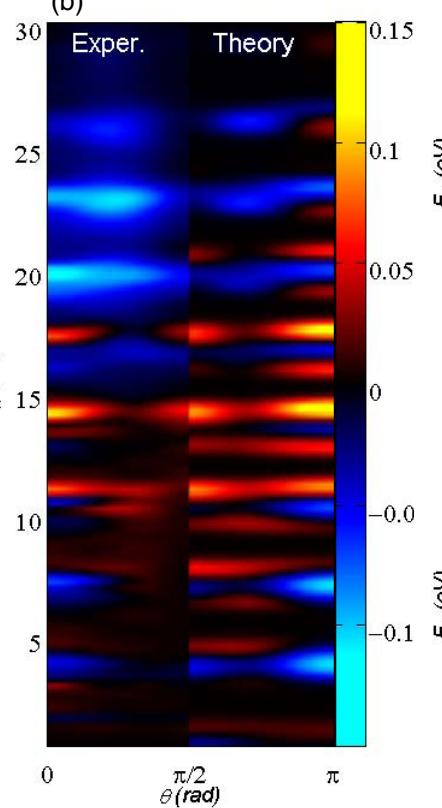

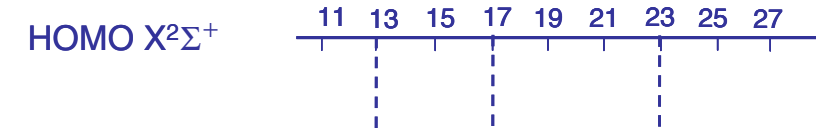

HOMO-1 A A $^{2} \Pi \begin{array}{llllllllll}11 & 13 & 15 & 17 & 19 & 21 & 23 & \text { \$5 } & 27\end{array}$

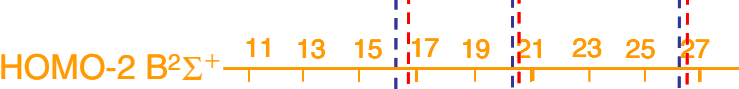

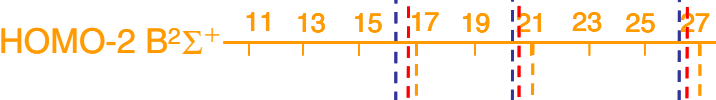

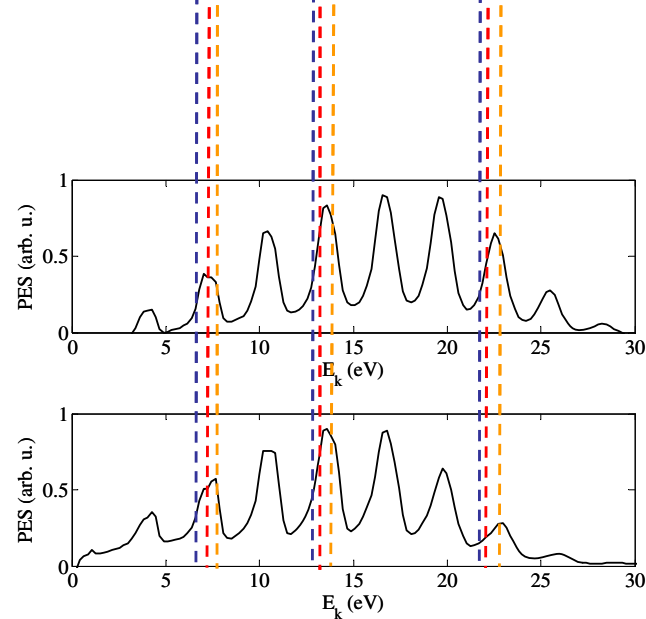

(c) $x^{2} \Sigma^{+}$

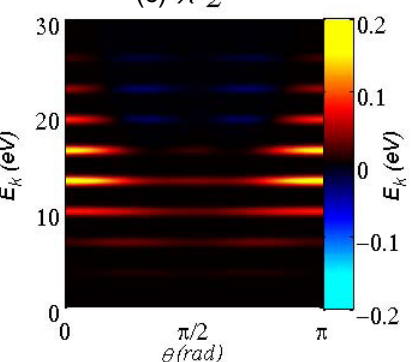

(d) $A^{2} \Pi$

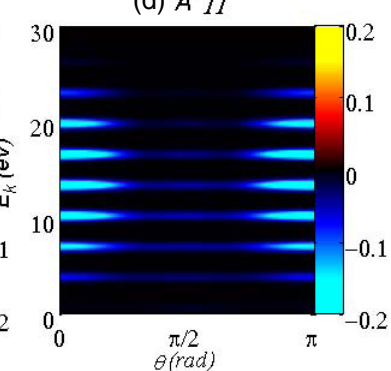

(e) $B^{2} \Sigma^{+}$

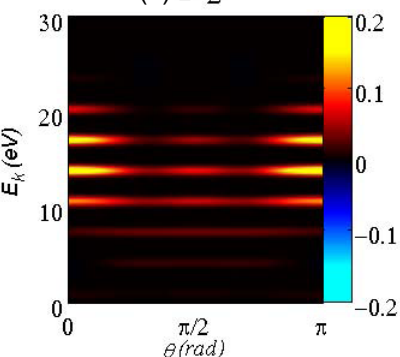

Figure 8. (a) Ionization of CO by the XUV pulse, involving contributions from harmonics $\mathrm{H} 11-\mathrm{H} 27$. The bottom-left panel shows the retrieve 2D slice through the 3D electron momentum distribution obtained after inversion of the experimental data. The bottom-right panel shows the integrated photoelectron spectra along (top) and perpendicular (bottom) to the laser polarization. (b) Angular and kinetic energy distribution of the difference of PADs obtained in aligned and anti-aligned CO molecules and comparison with theory. (c)-(e) The calculated evolution of the difference of PADs with respect to the kinetic energy for the three ionization channels observed in the experiment.

contributions highly overlap as shown in the photoelectron spectra in figure $7(\mathrm{a})$.

As for $\mathrm{O}_{2}$, a differential photoelectron momentum distribution was obtained by taking the difference between the PAD recorded at the maximum of alignment $\left(\Delta t_{\mathrm{IR}-\mathrm{XUV}}=\right.$ $8.6 \mathrm{ps})$ and one recorded under conditions of planar delocalization $\left(\Delta t_{\mathrm{IR}-\mathrm{XUV}}=8 \mathrm{ps}\right)$. A comparison of the numerical and experimental results is shown in figure $7(\mathrm{~b})$.
The calculation was performed considering a fixed internuclear distance of 1.09768 Angström in $\mathrm{N}_{2}$. Photoelectron kinetic energy-dependent changes in the difference of PADs are observed and well reproduced by the calculations which are individually shown for the three ionic states in figures 7(c)-(e). In the case of $\mathrm{N}_{2}$, one can see that ionization to the $\mathrm{X}^{2} \Sigma^{+}{ }_{\mathrm{g}}$ and $\mathrm{B}^{2} \Sigma^{+}$u final ionic states leads to a positive contribution in the difference of PADs whereas ionization to the $A^{2} \Pi_{u}$ has 

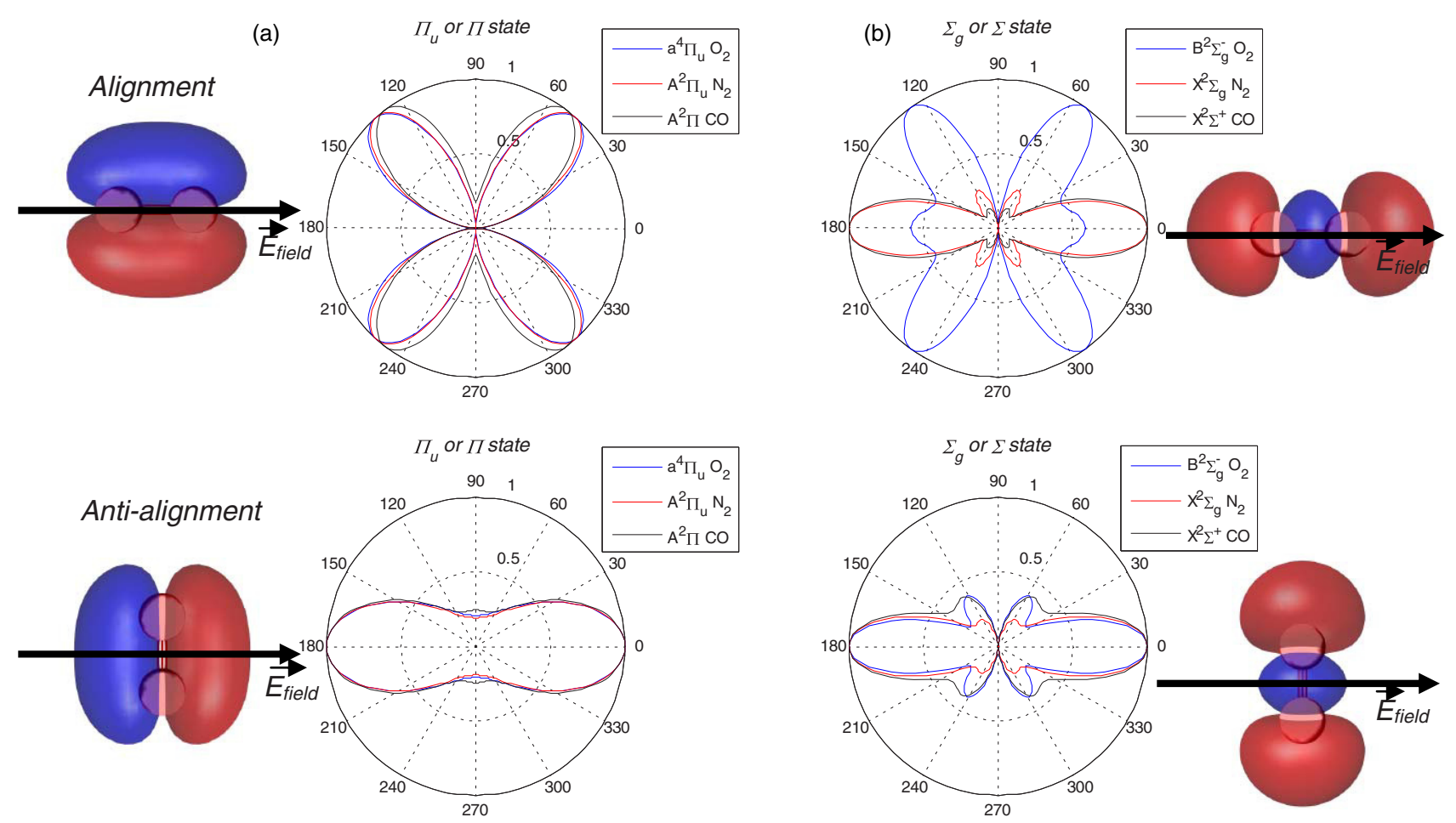

Figure 9. Calculated MFPAD for the ionization channel leading to (a) the final $\mathrm{a}^{4} \Pi_{\mathrm{u}}, \mathrm{A}^{2} \Pi_{\mathrm{u}}$ and $\mathrm{A}^{2} \Pi$ and (b) $\mathrm{B}^{2} \Sigma^{-}{ }_{\mathrm{g}}, \mathrm{X}^{2} \Sigma_{\mathrm{g}}$ and $\mathrm{X}^{2} \Sigma^{+}$ionic states of $\mathrm{O}_{2}, \mathrm{~N}_{2}$ and $\mathrm{CO}$ molecules, respectively. Top: the case of perfect alignment of the molecular axis along the laser polarization; bottom: the planar delocalization case.

a negative contribution. Starting from the $\Sigma^{+}$g ground state of $\mathrm{N}_{2}$, the symmetry of the total photoelectron + ionic state is restricted to $\Sigma^{+}{ }_{\mathrm{u}}$ for a parallel transition and to $\Pi_{\mathrm{u}}$ for a perpendicular transition. The positive difference of photoelectron yields that is experimentally observed in the $\mathrm{X}^{2} \Sigma^{+}{ }_{\mathrm{g}}$ and $\mathrm{B}^{2} \Sigma^{+}{ }_{\mathrm{u}}$ channels indicates a parallel transition and leads to the conclusion that the outgoing photoelectron has mainly $\sigma_{\mathrm{u}}$ symmetry in the case of the $\mathrm{X}^{2} \Sigma^{+}{ }_{\mathrm{g}}$ state and $\sigma_{\mathrm{g}}$ in the case of the $\mathrm{B}^{2} \Sigma^{+}{ }_{\mathrm{u}}$ state. Conversely, for the final ionic state $\mathrm{A}^{2} \Pi_{\mathrm{u}}$, where the allowed photoelectron symmetries are $\sigma_{\mathrm{g}}$ and $\pi_{\mathrm{g}}$ for perpendicular and parallel transitions, respectively, one can conclude from the sign of the observed difference of PADs that ionization to the $\mathrm{A}^{2} \Pi_{\mathrm{u}}$ cationic state leads to an outgoing photoelectron with mainly $\sigma_{\mathrm{g}}$ symmetry.

Using both the experimental and theoretical results, one can decompose the outgoing photoelectron wavefunction into partial waves characterized by $l$ and $\lambda$. For the $\mathrm{X}^{2} \Sigma_{\mathrm{g}}$ channel, symmetry considerations imply that the photoelectron can have $p \sigma_{\mathrm{u}}$ and $f \sigma_{\mathrm{u}}$ contributions. For this channel, we observe in figure 7(c) that below $10 \mathrm{eV}$ and above $22 \mathrm{eV}$, negative contributions appear in the difference of PADs which are due to the participation of $\mathrm{p} \pi_{\mathrm{u}}$ and $\mathrm{f} \pi_{\mathrm{u}}$ partial waves. This result is similar to the one observed in the ionization channels leading to the final $\mathrm{b}^{4} \Sigma^{-} \mathrm{g}$ and $\mathrm{B}^{2} \Sigma^{-} \mathrm{g}$ ionic states in $\mathrm{O}_{2}$. In the case of the $\mathrm{B}^{2} \Sigma^{+}{ }_{\mathrm{u}}$ final ionic state, one expects a strong $\mathrm{d} \sigma_{\mathrm{g}}$ partial wave with a preference for the electron to be ejected along and perpendicular to the laser polarization. This behaviour is well reproduced in our experiment, as well as in the calculation shown in figure 7(e). The $\mathrm{A}^{2} \Pi$ final ionic state should behave similar to the HOMO-2 ionization of $\mathrm{O}_{2}$. Indeed, one can see in figure $7(\mathrm{~d})$ that the difference of PADs is negative over the full photon energy range investigated, with a pronounced dip along and perpendicular to the laser polarization which is characteristic of a $\mathrm{d} \sigma_{\mathrm{g}}$ partial wave.

\section{CO}

Figure 8(a) shows the electron momentum distribution recorded for $\mathrm{CO}$ molecules after ionization with the XUV beam only. As for the other molecules, one can identify contributions from three distinct channels, namely the $\mathrm{X}^{2} \Sigma^{+}(14 \mathrm{eV}), \mathrm{A}^{2} \Pi(16.9 \mathrm{eV})$ and $\mathrm{B}^{2} \Sigma^{+}(19.7 \mathrm{eV})$ states [21, 22].

Figure 8(b) shows a comparison between the difference of PADs observed experimentally and computed theoretically using the procedure used for $\mathrm{O}_{2}$ and $\mathrm{N}_{2}$. A very good agreement is also observed in this case. The electronic structure of $\mathrm{CO}$ is very similar to $\mathrm{N}_{2}$ with the main difference being that $\mathrm{CO}$ is a heteronuclear molecule. However, since in our experiment the molecule is only aligned (and not orientated), no gerade/ungerade symmetry can be identified and we therefore expect a very similar result. Indeed, the three ionization channels lead to the same signature in the differential PAD that was previously observed for $\mathrm{N}_{2}$. Ionization to the $\mathrm{A}^{2} \Pi$ and $\mathrm{B}^{2} \Sigma^{+}$cationic states both are dominated by $\mathrm{d} \sigma_{\mathrm{g}}$ partial wave emission, with a strong negative contribution along and perpendicular to the laser polarization in the former case, and a strong positive contribution along and perpendicular to the polarization axis in the latter (figures 8(d) and (e)). The $\mathrm{X}^{2} \Sigma^{+}$channel also shows similar tendencies as the $\mathrm{X}^{2} \Sigma_{\mathrm{g}}$ channel of $\mathrm{N}_{2}$ with a strong modulation of 
the angular distribution of the ejected photoelectron with the photon energy. This effect can be attributed to the mixing of parallel $\sigma_{\mathrm{u}}$ and perpendicular $\pi_{\mathrm{u}}$ contributions together with the mixing between $p$ and $f$ partial waves that evolve with the kinetic energy.

\section{Discussion}

Comparing the three molecules, one can repeatedly recognize similar signatures in the difference of PADs. For instance, single photon ionization from a $\mathrm{X}^{2} \Sigma_{\mathrm{g}}\left(\mathrm{X}^{2} \Sigma\right.$ for $\left.\mathrm{CO}\right)$ state to a $А \Pi_{\mathrm{u}}(\mathrm{A} \Pi$ for $\mathrm{CO})$ state is consistently accompanied by the ejection of an electron with a dominant $d$ character. This is further demonstrated in figure 9(a), which shows a comparison between the MFPAD calculated in the case of perfect alignment for the $a^{4} \Pi_{u}, A^{2} \Pi_{u}$ and $A^{2} \Pi$ ionic states of $\mathrm{O}_{2}, \mathrm{~N}_{2}$ and $\mathrm{CO}$ molecules, respectively. These three channels have the same orbital shape and lead to a favoured electron emission at $45^{\circ}$ from the laser polarization.

A similar result is found when the $\mathrm{B}^{2} \Sigma^{-}{ }_{\mathrm{g}}, \mathrm{X}^{2} \Sigma_{\mathrm{g}}$ and $\mathrm{X}^{2} \Sigma^{+}$cationic states of $\mathrm{O}_{2}, \mathrm{~N}_{2}$ and $\mathrm{CO}$ are formed (see figure 9(b)). These three channels share a common orbital shape and lead to an electronic wave packet mainly composed of $\mathrm{p}$ (ejection along the laser polarization) and $\mathrm{f}$ (ejection along and at $60^{\circ}$ from the laser polarization) partial waves. It is also in these three channels that we have seen the strongest modification of the difference of PADs when increasing the photon energy. Calculations show that the modification of the $\mathrm{p} / \mathrm{f}$ partial wave ratio in this channel is not the main contribution responsible for the observed effect, but rather that a change in the symmetry of the outgoing electron from $\sigma$ to $\pi$ is responsible, meaning the ratio of parallel to perpendicular transition.

Figure 9 shows as well the photoelectron angular distribution in the case of anti-alignment of the molecular axis with respect to the laser polarization. For the two orbital shapes, we found as well a specific angular distribution for all three molecules.

\section{Conclusions}

In this study, we have shown the first measurements utilizing a novel short pulse XUV source to measure PADs from aligned molecules. It is shown that the PADs reflect the orbital symmetries of the ionization channels that are accessed in this photon energy range. Despite the modest degree of alignment achieved, the measured and calculated difference of PADs allows us to reveal quantitative information about the different molecular orbitals involved. Also the modifications of the PAD when increasing the photon energy reveal the influence of the molecular structure on the ejected photoelectron. It follows that the PADs from aligned molecules that we have measured reflect both the orbital symmetries of the ionization channels that are accessed and the onset of a scattering pattern related to the molecular field, and consequently, the molecular structure. This experiment paves the way towards the study of ultrafast electron and nuclear dynamics in small- and mediumsize molecular systems making use of the attosecond pulse structure of the XUV light that is produced by means of high-harmonic generation. Combining these tools together in a pump-probe arrangement should allow us to simultaneously probe attosecond electron [23, 24] and femtosecond nuclear dynamics [25] in real time.

\section{Acknowledgments}

This work is part of the research program of the 'Stichting voor Fundamenteel Onderzoek der Materie (FOM)', which is financially supported by the 'Nederlandse organisatie voor Wetenschappelijk Onderzoek (NWO)'. We acknowledge support from the European research council through the 'ATTOFEL' Marie Curie Initial training network. RRL acknowledges support from the Chemical Sciences, Geosciences and Biosciences Division, Office of Basic Energy Sciences, Office of Science, US Department of Energy and from the Robert A. Welch Foundation of Houston, TX, under grant no A-1020.

\section{References}

[1] Bandrauk A D, Manz J and Vrakking M J J 2009 Chem. Phys. 3661

[2] Sansone G et al 2010 Nature 465763

[3] Landers A et al 2001 Phys. Rev. Lett. 87 e013002

[4] Larsen J J, Sakai H, Safvan C P, Wendt-Larsen I and Stapelfeldt H 1999 J. Chem. Phys. 1117774

[5] Rosca-Pruna F and Vrakking M J J 2001 Phys. Rev. Lett. 87153902

[6] Holmegaard L et al 2010 Nature Phys. 6428

[7] Meckel M et al 2008 Science 3201478

[8] Hockett P et al 2009 Phys. Rev. Lett. 102253002

[9] Kelkensberg F, Rouzée A, Siu W, Gademann G, Johnsson P, Lucchini M, Lucchese R R and Vrakking M J J 2011 Phys. Rev. A 84051404

[10] Ghafur O, Siu W, Johnsson P, Kling M F, Drescher M and Vrakking M J J 2009 Rev. Sci. Instrum. 80033110

[11] Lépine F et al 2007 J. Mod. Optics 54953

[12] Renard V et al 2004 Phys. Rev. A 70033420

[13] Suzuki I H, Weigold E and Brion C E 1980 J. Electron Spectrosc. Relat. Phenom. 20289

[14] Lafosse A et al 2002 J. Chem. Phys. 1178368

[15] Stratmann R E and Lucchese R R 1995 J. Chem. Phys. 1028493

[16] Stratmann R E, Zurales R W and Lucchese R R 1996 J. Chem. Phys. 1048989

[17] Lucchese R R, Lafosse A, Brenot J C, Guyon P M, Houver J C, Lebech M, Raseev G and Dowek D 2002 Phys. Rev. A 65020702

[18] Lucchese R R, Takatsuka K and Mckoy V 1986 Phys. Rep. 131147

[19] Kendall R A, Dunning T H and Harrison R J 1992 J. Chem. Phys. 966796

[20] Dunning T H 1989 J. Chem. Phys. 901007

[21] Plummer E W, Gustafsson T, Gudat W and Eastman D E 1977 Phys. Rev. A 152339

[22] Turner D W, Baker C, Baker A D and Brundle C R 1970 Molecular Photoelectron Spectroscopy (New York: Wiley-Interscience)

[23] Breidbach J and Cederbaum L S 2005 Phys. Rev. Lett. 94033901

[24] Remacle F and Levine R D 2006 Proc. Natl Acad. Sci. 1036793

[25] Bisgaard C Z et al 2009 Science 3231464 